\title{
Concentration of zinc ions in perchlorate medium by a menbrane-gel using an acid extractant (DEHPA)
}

\author{
L. Belhadji ${ }^{1}$, F. Belkacemi ${ }^{2}$, and D.E. Hadj-Boussaad ${ }^{3}$ \\ ${ }^{1}$ Laboratoire d'analyse fonctionnelle des procédés chimiques (LAFPC), département de chimie industrielle, Faculté des \\ sciences de l'ingénieur, Université Saad Dahleb de Blida, route de Soumaa B.P 270, 09000 Blida, Algeria \\ ${ }^{3}$ Laboratoire des Applications énergétiques de l'hydrogène, département de médecine, Faculté des Sciences de \\ l'Ingénieur, Université Saad Dahleb de Blida, Route de Soumâa, B.P 270, 09000 Blida, Algeria
}

\begin{abstract}
Recent decades, it is an awareness of the importance of ecological balance in the environment, balances threatened by industrial pollution. A new spirit presides we seek to minimize pollution of receiving waters. The present work is to study the liquid-gel-extraction of zinc ions in perchlorate medium by an acid extractant: the di (ethyl-2 hexyl) phosphorique acid, or DEHPA. Two types of polymers were used as supports of solvent extraction: a polybutadiene rubber cross-linked respectively with $0.1 \%$ dicumyl peroxide and $0.4 \%$ dicumyl peroxide, vulcanized at $160^{\circ} \mathrm{C}$, one is most cross-linked than the other. The liquid-gel extraction is based on the principles of the liquid-liquid extraction.
\end{abstract}

\section{Introduction}

Membrane separation processes are used to separate and concentrate especially molecules or ionic species in solution, for separating particles or microorganisms in suspension in a liquid.

These separation methods must now be considered as advanced technology, performance and cost. Among these means, it is the liquid-gel based on the same as those of the liquid-liquid extraction.

The liquid-liquid extraction has sometimes problems which complicate the emulsion phase separation and result in losses of extractant due to the solubilization of the latter as well as the training effect in aqueous phase. Also, for this eventuality, several researchers have proposed to absorb the organic phase extractant to solid supports such as polymers.

Hale [1] Small [2] worked on polystyrene Braun [3, 4] have done on polyurethane foams and Baghai [5] silicone rubber.

Brunette et al. [6, 7] studied the liquid-gel metal ions. The liquid-gel has the advantage of combining the favorable effects of the liquid-liquid extraction and those of the liquid-solid extraction is a technique that can be implemented to solve analytical problems but also to separate and recover metal elements of economic interest (secondary materials), or to preserve the environment, [8$10]$.

In this work we propose to implement a particular type of membranes, membrane liquid-gel and adapt to the reduction and concentration of zinc from hydrometallurgical solutions and a thermodynamic study performed to see the form of the complex formed. In most thermodynamic studies on the reactions of liquid- liquid extraction of zinc complex shows that the extract is generally shaped ZnI2, Rice and Smith in 1975 [11] and Ajawin All in 1983 [12] Sastre and Muhammed [13] and finally Apaccio and Muhamed [14] showed that the species extracted in the case of zinc are generally [ $\mathrm{ZnI} 2$, 2HL] DEHPA or both at once. These also depending on the type of diluent used [15].

\section{Experimental metodology}

Our membrane is a polymer which, in contact with a solvent, undergoes an increase in volume "swelling". The latter is due to the expansion of the network, caused by the gradual penetration of solvent molecules between the macromolecular chains of the polymer [16].

The experimental work involves three steps:

-The first step is to prepare the gel membrane from the polymer and solvent chosen.

-The second stage will be devoted to liquid-gel menbrane zinc extraction. We have undertaken to use a specific extractant metals an extractant acid chelate: acid di (2-ethyl hexyl) phosphoric acid (DEHPA).

- Complex nature of extract thermodynamic study.

\subsection{Preparation of gel extractant}

In a first stage, the amount of extractant in the gel is determined by simple measurement of the weight of polymer before and after swelling, the swelling ratio. The latter depends on the dwell time and the solubility of the polymer. In a second step, the solvent and extractant do not necessarily penetrating homogeneously in the polymer; the exact composition of the extractant in the 
gel is determined by the relative penetration of extractant over time.

In our work, the extractant is the gel swollen polymer in an organic solution DEHPA extractant dissolved in a solvent; kerosene at various concentrations of $0.02,0.2$ and $2.97 \mathrm{M}$.Two types of polybutadienes were studied $\mathrm{O} .4$ and $0.1 \%$ dicumyl peroxide cured at $160^{\circ} \mathrm{C}$, respectively:

- One for 15 minutes, designated Br1.

- One for 5 minutes, designated $\mathrm{Br} 2$.

\subsection{Swelling in pure solvents}

Three types of solvents previously washed with distilled water have been the subject of our study:

- An aromatic: Toluene

- A chlorine, chloroform

- An oil cut: Kerosene purified beforehand

Samples of different types of polymers were immersed in a vial containing each of the three solvents mentioned above. All bottles were placed in thermoregulated bath at $25^{\circ} \mathrm{C}$; the gels obtained are weighed during specific time intervals

\subsection{Recovery of $\mathrm{Zn}^{2+}$ ions by different systems}

Samples of reticulated of polymer as already definite are inflated initially in the pure DEHPA in a first stage then respectively in solutions of diluted DEHPA with 0,$02 ; 0,2$ and 2,97 $\mathrm{M}$ in the kerosene during 30 minutes. The gel thus obtained is weighed then plunged in $5 \mathrm{ml}$ of a solution of zinc to $0,15.10-2 \mathrm{M}$ in $(\mathrm{Na}, \mathrm{H}) \mathrm{ClO} 41 \mathrm{M}$ with $25^{\circ} \mathrm{C}$ under agitation.

Samples are taken over time until the extraction equilibrium is reached. Zinc in aqueous phase is determined by UV spectrometry at a wavelength $533 \mathrm{~nm}$. [17].

\subsection{Thermodynamic study}

The organic phases are obtained by swelling of crosslinked $\mathrm{Br}_{2}$ same masses, in a solution of DEHPA in kerosene at various concentrations from 0.2 to $1 \mathrm{M}$. After the gels obtained, they are immersed in each $5 \mathrm{ml}$ of the aqueous solution zinc $0.1510^{-2} \mathrm{M}(\mathrm{Na}, \mathrm{H}) \mathrm{ClO} 4,1 \mathrm{M}, \mathrm{pH}$ $=1$ with stirring at $250^{\circ} \mathrm{C}$.

\section{Results and discussion}

\subsection{Preparation of gel extractant}

\subsubsection{Swelling}

The maximum time of swelling of polymers is determined by the weighed successive ones, carried out according to time until obtaining a profit in stationary weight; this last corresponds to the balance of swelling [18].

The results giving the rates of swelling of two polymers are as follows (Table 1).
Table 1. Rate of swelling mass polymer.

\begin{tabular}{|l|c|c|c|c|}
\hline \multicolumn{1}{|c|}{ Solvants } & $\begin{array}{c}\tau \\
\left(\mathrm{BR}_{1}\right)\end{array}$ & $\begin{array}{c}\text { Temps de } \\
\text { gonflement } \\
\text { à l'équilibre } \\
(\mathrm{min})\end{array}$ & $\begin{array}{c}\tau \\
\left(\mathrm{BR}_{2}\right)\end{array}$ & $\begin{array}{c}\text { Temps de } \\
\text { gonflement } \\
\text { à l'équilibre } \\
(\text { min })\end{array}$ \\
\hline Chloroforme & 4.36 & 240 & 2.8 & 180 \\
\hline Toluène & 4.0 & 240 & 2.3 & 180 \\
\hline Kérosène & 4.6 & 120 & 2.5 & 180 \\
\hline DEHPA & 1.3 & 20 & 1.22 & 35 \\
\hline
\end{tabular}

Nodes (or crosslinking) of the two polymers favor the trapping of molecules in question. Therefore, $\mathrm{Br}_{1} \mathrm{Br}_{2}$ is more than cured, it provides less likely to engage Site branched forms as indicated in Table 1, and, for example, the rate for the system is relatively Kerosene $\mathrm{Br}_{1} 2$ times more than the system Kerosene/ $\mathrm{Br}_{2}$. Increasing the degree of crosslinking is accompanied by a decrease in swelling

\subsubsection{Penetration of the extractant in the polymer}

It is the quantity of extractant which would have penetrated in the polymer which interests us more in the direction or one would have a better complexation of metal by organophosphorés (DEHPA) in polymer. To determine the relative penetrations of extractant in polymer, one will determine the concentration of extractant in freezing according to time by using the balance of the masses [19]. The following curve was obtained; given the results, it appears that the diluent kerosene and solute DEHPA penetrate evenly throughout the gel. The concentration of acid in the gel is $86 \%$ of its initial value in the organic phase. Based on these results, we have chosen for the rest of our work, the systems showed good penetration extractant: Kerosene and $\mathrm{Br}_{1}$

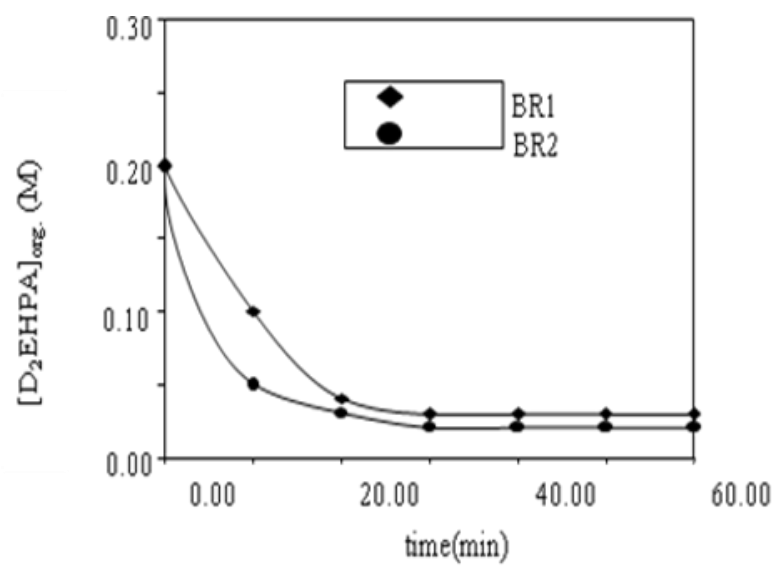

$$
\text { Figurel Variation of }[D E H P A]_{\mathrm{ogg}} \text { in the solution of swelling }
$$
system $\mathrm{Br}_{1}$ and $\mathrm{Br}_{2} / \mathrm{DEHPA} / \mathrm{Kérosène.}$

\subsection{Recovery of $\mathrm{Zn}^{2+}$ by different systems}

The graphical representation of the kinetics and efficiency of extraction of zinc is illustrated by the following figures 2 and 3 . 


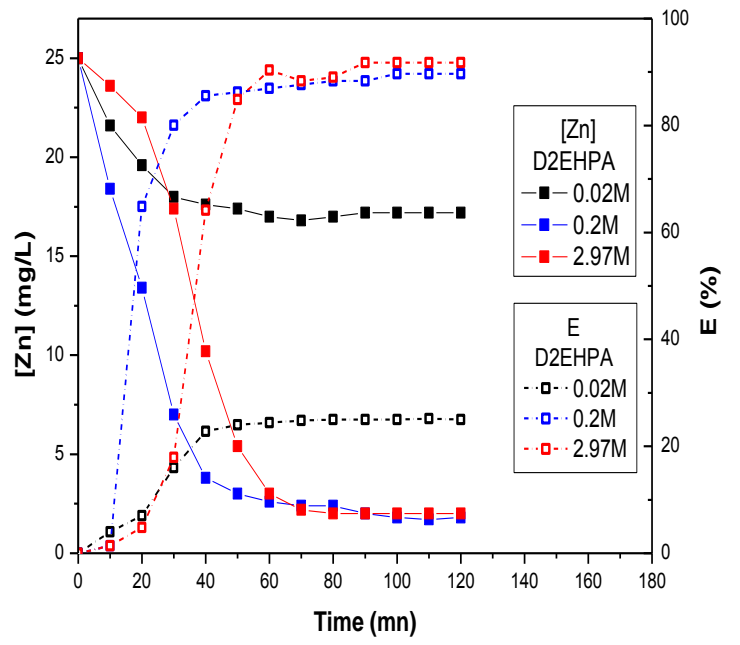

Figure 2. Kinetics and yield extraction of zinc ions by the system BR1/DEHPA/Kerosene.

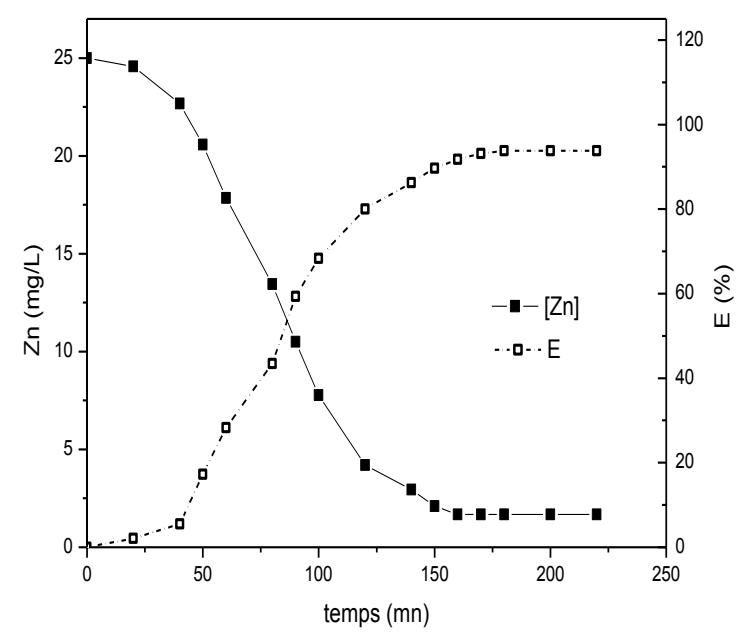

Figure 3. Extraction of zinc liquid-gel the system Br1/DEHPA pur.

\subsection{Thermodynamic study of extraction the liquid- gel}

Complex nature of the extract in the case of acid extractants HA. The extraction by extractants acids occurs by the following mechanism:

$$
M^{n+}+n H A \quad \leftrightarrows \overline{M_{n}}+n^{+}
$$

$\mathrm{K}=\frac{\mathrm{HA}_{\mathrm{n}} \cdot \mathrm{H}^{+}}{\mathrm{1}^{\mathrm{n}+} \cdot \mathrm{HA}_{-}^{\overline{\mathrm{n}}}}$

The logarithm of the equilibrium constant gives:

$$
\log \mathrm{K}=\mathrm{npH}+\mathrm{n} \log \overline{\mathrm{A}}_{-}^{-} \log \mathrm{D}
$$

Where

$$
\log \mathrm{D}=\log \mathrm{K}+\mathrm{npH}+\mathbf{n} \log \overline{\mathrm{A}}^{-}
$$

From this expression, it is possible to have an idea about the formula of the complex extract. Indeed, the coefficient $\mathrm{n}$ gives the number of molecules extractant put into play, and the slope of $\log \mathrm{D}=\mathrm{f}(\log [\mathrm{HA}])$ at constant $\mathrm{pH}$ :

\section{$\mathrm{Zn} 2+2 \mathrm{n} \overline{\mathrm{HA}} \leftrightarrows \mathrm{Zn} \mathrm{A} 2+2 \mathrm{H}+$}

To determine the different zinc complex can be formed in the liquid-gel, we plotted the variations of the logarithm of the distribution coefficient of the logarithm of the concentration of the extractant, it was found.

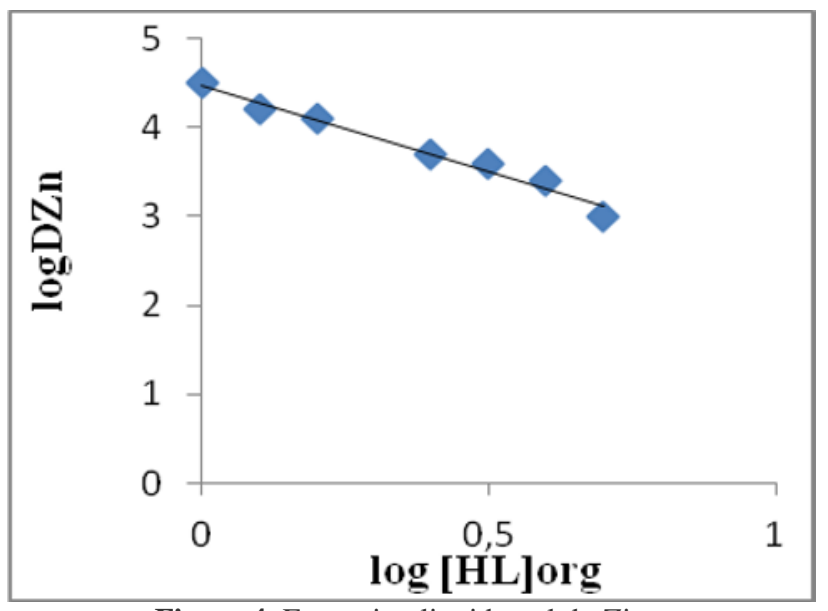

Figure 4. Extraction liquide-gel du Zinc.

The experimental curve obtained by plotting the logarithm of the distribution variation of the zinc from the aqueous phase and the organic phase as a function of the logarithm of the concentration of the extractant.

System for liquid-gel experimental curve is a straight line of slope 1, 93 suggest that this organic phase is formed, a complex whose report (ligand/metal) is close to 2 , it is assumed that the complex Formed is of type $\mathrm{ZnL}_{2}$ ou $\mathrm{Zn}\left[\left(\mathrm{C}_{8} \mathrm{H}_{17} \mathrm{O}\right)_{2} \mathrm{P}(\mathrm{O}) \mathrm{O}^{-}\right] 2$.

\section{Conclusion}

The impregnation of polybutadiene crosslinked with an organic solution for the extraction of zinc shows good penetration of the extractant in the gel. The use of gel swollen with polybutadiene around in acid solutions (DEHPA) and in solutions of the same acid diluted in kerosene, extraction of zinc from solutions initially set to $0,15.10-2 \mathrm{M}$ perchlorate in medium $\mathrm{M}$ by the system in mid Br1/DEHPA perchlorate provides an extraction yield of about $83 \%$.

The use of gels swollen polybutadiene type $\mathrm{Br}$ edge solutions in acid (DEHPA) and in solutions of the same acid diluted in kerosene has enabled us to handle large volumes of aqueous phase loaded with low volumes of organic phase without the problem of phase separation arises. 
The study of a part of thermodynamics showed the existence of the species extracted into the organic phase, which is the type $\mathrm{ZnL} 2$.

\section{References}

1. D.K Hale, National Research. British Patent, 1955 BR. 738, 500

2. H. Small, the Dow chemical company, U.S.Patent, Sept.1963,102-782

3. T. Braun, M.N. Abbas, A. Vertes, Radiochem. Letters 54 (1982)

4. S. Torok, T. Braun, P. Van Dick, R. Van Grieken, XRay Spectrometry 15-1-7 (1986)

5. A. Baghai, H.M. Bowen, Analyst 101661 (1976)

6. J.P. Brunette, E.H. Riffi, M.J.F. Leroy, P. Mallo, G. Waton, M. Prevost, Solvent Extr. Ion Exch. 51017 (1988)

7. J.F. Loret, J.P. Brunette, M.J.F. Leroy, S.J. Candau, M. Prevost, Solvent Extr.Ion Exch. 6585 (1988)

8. H. Reinhard, H. Ottertun, T. Troeng, Inst.chem.Eng.Symp. Ser. 41 W1 (1975)

9. D.S. Flett, Chem. Technol. Biotechnol. 29258 (1979)

10. E.D. Nogueira, J.M. Regife, P.M. Blythe, Chem. Ind. London 263 (1980)

11. N.M. Rice and M.R. Smith, Chim. Biotechnol. 25379 (1975)

12. L.A. Ajawin, E.S.P. Ortiz, H. Sawistowkih, Res. Des. Chem.Eng. 6162 (1983)

13. A.M. Sastre and M. Muhammed, Hydrometallurgy 12 177 (1984)

14. A. Apacio, and M. Muhammed, Hydrometallurgy 21 3 5-299 (1989)

15. Z.C. Li, Furst, H., Wand Renun., Hydrometallurgy 16 231 (1986)

16. F. Katzonevas, Technique de l'ingénieur, Elastomère A 7705 (1983)

17. J. Rodier, Analyse de l'eau, Dunod, 8ème Ed. (1996)

18. N. Taoualit, D.E. Hadj-Boussaad, Desalination 273 (2002)

19. N. Taoualit, D.E.Hadj-Boussaad, Desalination 193 321 (2006) 